\title{
Un enfoque participativo para la formación docente continua en la Educación en Ciencias Naturales, Ambiental y en Salud
}

\author{
Ana Dumrauf \\ Grupo de Didáctica de las Ciencias, Instituto de Física de Líquidos y Sistemas Biológicos (IFLYSIB, \\ UNLP-CONICET). Departamento de Ciencias de la Educación, Facultad de Humanidades y Ciencias de \\ la Educación, Universidad Nacional de La Plata (UNLP), Argentina. \\ adumrau@iflysib.unlp.edu.ar \\ ORCID: bttps:/ / orcid.org/0000-0002-4856-787X \\ Silvina Cordero \\ Grupo de Didáctica de las Ciencias, Instituto de Física de Líquidos y Sistemas Biológicos (IFLYSIB, \\ UNLP-CONICET). Departamento de Ciencias de la Educación, Facultad de Humanidades y Ciencias de \\ la Educación, Universidad Nacional de La Plata (UNLP), Argentina. \\ cordero@iflysib.unlp.edu.ar \\ ORCID: https:/ / orcid.org/0000-0002-0254-041X
}

[Recibido: 4 Febrero 2019. Revisado: 16 Julio 2019. Aceptado: 17 Agosto 2019]

Resumen: Se presentan características y fundamentos de un enfoque de formación docente continua en Educación en Ciencias Naturales, Ambiental y en Salud elaborado a partir de una experiencia de 3 años con docentes de distintos niveles educativos. Se analiza cómo y en base a qué fundamentos se desarrolló un proceso de formación e investigación participativa que aportó a la transformación de prácticas de enseñanza. El enfoque permitió articular la investigación educativa con la realidad escolar a través de la producción conjunta de conocimientos. Promovió cambios en las prácticas docentes respecto de la lectura de lo real y las relaciones sociales al estimular la construcción y análisis colectivos de propuestas y la participación estudiantil. Finalmente, fue valorado el papel de la praxis a través de la producción de acciones exploratorias. Los cambios logrados se orientaron a transformar la realidad educativa desde una intencionalidad emancipatoria.

Palabras clave: Formación docente continua; Investigación participativa; Educación en Ciencias Naturales, Ambiental y en Salud

A participatory approach for in-service teacher instruction in Natural Sciences, Environmental and Health Education

Abstract: Features and grounds of an approach of in-service teacher instruction in natural sciences, environmental and health education, elaborated from an experience of 3 years with teachers from different educational levels, are presented. It was an interest to analyze how and on what basis was developed a process of training and participatory research that contributed to the transformation of teaching practices. The approach allowed joint educational research with school reality through the joint production of knowledge. It promoted also changes in teaching practices referred to the reading of the real and social relations by stimulating the collective construction of proposals and analysis and student participation. Finally, the role of praxis was appreciated through the production of exploratory actions. The changes made were oriented to transform the educational reality from an emancipatory intention.

Keywords: In-service teacher instruction; Participatory research; Natural Sciences, Environmental and Health education.

Para citar este artículo: Dumrauf. A y Cordero. S. (2020) Un enfoque participativo para la formación docente continua en la Educación en Ciencias Naturales, Ambiental y en Salud. Revista Eureka sobre Enseñanza y Divulgación de las Ciencias 17(1), 1602. doi: 10.25267/Rev_Eureka_ensen_divulg_cienc.2020.v17.11.1602 


\section{Introducción}

Desde hace varios años, nuestro recorrido como investigadoras fue configurándose en torno a la preocupación compartida en el campo por la distancia existente entre las producciones de la investigación educativa y las prácticas docentes cotidianas (Zeichner 1998; Copello Levy y Sanmartí Puig 2001; Oliva 2012, 2011; Cordero y Mengascini 2016; entre otros). Una de las claves para promover cambios en esta situación es la renovación de enfoques en la formación docente (Copello Levy y Sanmartí Puig 2001; Delord, Porlán y Harres 2017).

Específicamente, respecto de la formación docente en ejercicio, son necesarios planteamientos innovadores para responder a las características propias de esta compleja profesión (Lupion Cobos y Martín Gámez 2016). Como condiciones que favorecen la innovación y el cambio en la enseñanza de las ciencias, los estudios identifican la importancia de: la consideración de concepciones y prácticas docentes; la participación voluntaria y deseada en los procesos de formación; el trabajo cooperativo; la reflexión colectiva y la pertenencia a grupos de innovación que garanticen el sostenimiento de las transformaciones logradas; y el intercambio horizontal entre docentes e investigadores/as (Delord et al. 2017). Estas condiciones darían un nuevo marco al desarrollo profesional docente.

En Argentina, entre 2005-2015, algunos estudios identifican desplazamientos de las propuestas formativas hacia modelos de desarrollo profesional -al menos discursivamente- y una incipiente recuperación de la centralidad de la política frente a la lógica economicista y tecnocrática de los '90 (Feldfeber 2011). Sin embargo Menghini, Díaz, Gardié e Iriarte (2011) sostienen que las políticas educativas en general, y las de formación docente en particular, no fueron muy distintas de las previas, debido a su fuerte centralización, su oferta a través de una sumatoria de programas sobre distintos temas, y a ser meramente declarativas de la participación docente desde una visión instrumental.

En este contexto, entre 2008 y 2010, implementamos el proyecto Investigación colaborativa para la reconstrucción de prácticas y la innovación en educación en Ciencias Naturales que ofreció un espacio de formación en ejercicio a partir de valorar los saberes docentes y reconocer la complejidad de su práctica. Por medio de la ampliación del universo conceptual y didáctico de los/as docentes y de la investigación participativa, apuntó a reflexionar sobre la práctica, construir en conjunto propuestas para su transformación y analizarlas colaborativamente. Consideramos que, en este proceso, los saberes docentes se articularían con el saber experto y las experiencias desarrolladas por otros/as colegas, y se nutrirían de la teoría y la investigación educativa. A través de la práctica y teorizando sobre ella, antes, en y después de la acción, elaboramos un enfoque para la formación docente continua en el campo de la Educación en Ciencias Naturales, Ambiental y en Salud, cuyas características desarrollamos en este trabajo. En su construcción se buscó responder cómo y en base a qué fundamentos, se podría llevar a la práctica un proceso de formación e investigación participativa, que fuera sostenido y favoreciera la transformación de las prácticas docentes. En este sentido, este análisis se enmarca en lo que Romera-Iruela (2011) denomina "artículos sobre modelos formativos" (p. 609).

Localizamos nuestra investigación en el campo de estudios en/sobre formación docente (Messina 1999; Achilli 2004) y, específicamente, a) buscamos identificar características del proceso formativo, promotoras de transformaciones en las prácticas de enseñanza de docentes de Ciencias Naturales de diversos niveles educativos y b) delineamos sus fundamentos teóricometodológicos a la luz de la investigación-acción participativa (a partir de aquí IAP).

A continuación presentamos los referentes teóricos que dan marco a este estudio. Luego describimos la experiencia y nuestra metodología de análisis, presentamos las características 
del enfoque que promovió cambios en las prácticas de enseñanza de los grupos participantes, y revisamos sus fundamentos atendiendo a los elementos claves de la IAP propuestos por Kemmis y McTaggart (2005).

\section{Referentes teóricos}

En los últimos años parece existir un consenso respecto de que las investigaciones que fortalecen el desarrollo profesional docente e influyen en sus prácticas son las realizadas por y con docentes, en equipos interdisciplinarios e interniveles, en los que el profesorado es coproductor y agente de cambio en la resolución de problemas que les afectan en sus clases (Vázquez-Bernal y Jiménez Pérez 2013). Pacca y Villani (2000), al investigar colectivos docentes, señalan que las redes proporcionan motivación de forma continua y renovada; y que la presencia de investigadores/as en ellas promueve diálogo entre teoría y práctica y colaboración horizontal entre universidad y escuela. En ese diálogo tanto prácticas educativas como investigaciones se ven enriquecidas (Tardif y Zourhal 2005). Como dicen Delord et al. (2017, p. 661) "Hacer investigaciones para la escuela requiere de la participación de los sujetos a quien se supone irán destinadas las conclusiones". En este marco, Vázquez-Bernal y Jiménez Pérez (2013) reconocen que la línea de los programas de Investigación-Acción ha sido fructífera para la formación continua y la producción de conocimiento con el profesorado de ciencias.

Para transformar las prácticas docentes, a nuestro criterio, son necesarios procesos de trabajo conjunto a fin de apropiarse de los sentidos de la acción educativa, tomar decisiones e intervenir en función de los sentidos asumidos. Estos procesos, promovidos desde una intencionalidad emancipatoria, pretenden "un movimiento colectivo e individual, de liberación consciente y de superación de las formas de alienación material y simbólica" (Loureiro 2003, p. 22). Compartiendo esta intencionalidad, Kemmis y McTaggart (2005) valorizan la participación de todas las personas involucradas y delinean y fundamentan una línea de IAP. Acuden a la Teoría de la Acción Comunicativa de Habermas $(1984,1996)$, para caracterizar sus elementos claves: el empoderamiento de las/os actores, el papel de lo colectivo, el rol del/la facilitador/a y el dualismo investigación-acción.

El empoderamiento logrado en la IAP nombra un proceso en el cual personas y grupos se comprometen auténticamente, reconociendo y respetando diferencias, en la toma de decisiones legítimas (porque han participado en ellas abierta, genuina y libremente comprometidos con la comprensión mutua, el acuerdo intersubjetivo y el logro del consenso para la acción). El poder comunicativo desarrollado en esferas públicas a través de la acción comunicativa y el discurso público constituyen, desde esta postura, las bases del empoderamiento. Dichas acciones y discursos se fundamentan en decisiones evaluadas como legítimas, justas y adecuadas a su realidad (Kemmis y McTaggart 2005). Sumamos a este planteo la importancia atribuida al ejercicio de la participación directa en la construcción y el sostenimiento del empoderamiento por perspectivas latinoamericanas de la IAP (Hoyos Vásquez 1998).

En su reflexión sobre lo colectivo, Kemmis y McTaggart (2005) sostienen que los grupos formados para implementar proyectos de IAP deben pensarse como espacios abiertos e inclusivos, constituidos para crear condiciones para la acción comunicativa libre y el discurso público. Su fin es atender situaciones de irracionalidad, injusticia e insatisfacción experimentadas en circunstancias particulares. Estos autores focalizan en el papel del/a facilitador/a en los proyectos de IAP. Reconociendo la existencia de asimetrías en los colectivos involucrados, caracterizan su rol como coparticipante que contribuye con su conocimiento y experticia. Señalan además que en diferentes momentos, distintos/as 
participantes pueden asumir ese papel en un mismo colectivo. Consideran que se trata de un rol que no debe ser entendido como un agente externo, que ofrece orientación técnica, sino como alguien que tiene como objetivo establecer o apoyar un trabajo colaborativo en el cual las personas se involucren en acciones exploratorias.

Finalmente, estos autores cuestionan la comprensión de manera separada de la investigación y la acción, entendiéndolas como convergentes en acciones comunicativas orientadas hacia acciones exploratorias. El espacio generado para la acción comunicativa en la IAP crea oportunidades para adoptar una mirada reflexiva y exploratoria respecto de qué hacer, sabiendo que la práctica será modificada a la luz de lo que se aprenda del análisis cuidadoso del proceso y sus consecuencias. Según Kemmis y McTaggart (2005, p. 300) "la acción comunicativa no es solo reflexión o práctica reflexiva [...] sino también acción realizada con el propósito principal de aprender de la experiencia a través de la observación cuidadosa de sus procesos y consecuencias. Es deliberadamente diseñada como una exploración de las maneras de hacer las cosas en una situación particular y en un momento histórico particular. Es diseñada para ser acción exploratoria" (traducción nuestra).

\section{Descripción de la experiencia y metodología de análisis}

Nuestra propuesta de desarrollo profesional implicó la implementación de un triple proceso de formación docente, transformación de las prácticas educativas e investigación participativa. Para elaborarla constituimos un equipo coordinador interdisciplinario e interinstitucional, con 5 integrantes de una institución de formación inicial de docentes (Instituto Superior de Formación Docente $\mathrm{N}^{\mathrm{o}} 168$, a partir de aquí ISFD ${ }^{1}$ ) y 3 del ámbito universitario (Universidad Nacional de La Plata, 2 de ellas, autoras de este artículo). A este equipo sumamos, en actividades específicas, colaboradoras/es de ambas instituciones.

Las/os participantes en la propuesta (convocados/as de manera voluntaria en su horario laboral) fueron docentes en ejercicio en establecimientos de gestión estatal de niveles Primario, Secundario y Superior próximos al ISFD. En total 168 docentes asistieron al menos a un encuentro de formación, 52 participaron regularmente del proceso; 39 diseñaron e implementaron propuestas didácticas transformadoras, y 29 completaron la instancia de análisis de la propia práctica en 3 años de trabajo. Las/os participantes provenían de 38 escuelas primarias; 6 secundarias y 2 ISFD. La dificultad del sostenimiento de un proceso extenso constituyó una de las causas de desgranamiento del grupo. Solo docentes de nivel primario finalizaron todo el trayecto, incluyendo la presentación pública de sus análisis, probablemente gracias a la posibilidad de concentración y continuidad de su tarea docente en solo una o dos escuelas.

En los 3 años de trabajo generamos diversos espacios de vinculación: encuentros generales de formación ( 9 en total de 6 horas cada uno), reuniones del equipo coordinador, de grupos docentes entre sí y del equipo coordinador con grupos docentes. Los primeros 4 encuentros ( $1^{\text {er }}$ año) abordaron temáticas generales respecto de la Educación en Ciencias Naturales (cuestiones epistemológicas, didácticas y vinculadas al análisis de la propia práctica); la evaluación conjunta del proceso vivido, la conformación de grupos de trabajo docente y la delimitación de tópicos curriculares para elaborar propuestas didácticas transformadoras de las prácticas. En el $2^{\mathrm{do}}$ año también se implementaron 4 encuentros sobre temáticas específicas seleccionadas a fin de abarcar los contenidos conceptuales y metodológicos básicos para el desarrollo de las propuestas didácticas (Educación Ambiental y enseñanza por proyectos; Educación para la Salud Sexual y modalidad de taller; y, dentro de la Física, luz y óptica, y

${ }^{1}$ En Argentina la formación docente inicial para los niveles primario, secundario y superior no universitario se desarrolla, mayoritariamente, en este tipo de instituciones de jurisdicción provincial. 
abordajes experimentales). En el último encuentro se socializaron las propuestas didácticas implementadas y evaluamos el camino recorrido. Durante ese año, se afianzaron los grupos docentes, redefinieron los tópicos a abordar, diseñaron, pusieron en práctica y registraron la implementación de las propuestas áulicas. Como equipo coordinador acompañamos su trabajo virtualmente, en reuniones en el ISFD y en las instituciones de procedencia de las/os docentes. Procuramos así una instancia personalizada de diálogo y conocimiento individual y contextual, en un espacio más propio y distendido y, en ese sentido, facilitador de la explicitación de dificultades e incertidumbres y promotor de vínculos de confianza. En el $3^{\text {er }}$ año continuamos acompañando a los grupos en el análisis de sus producciones; e implementamos un encuentro final de socialización de procesos y resultados grupales. Otros espacios de encuentro fueron, durante el $2^{\mathrm{do}}$ y $3^{\text {er }}$ años, las reuniones de trabajo independiente de cada grupo docente y el intercambio espontáneo entre ellos. Finalmente, desarrollamos las $1^{\text {eras }}$ Jornadas de Investigación Participativa en Educación en Ciencias Naturales, Ambiente y Salud (JIPE).

Como resultado del trabajo, se formaron 15 grupos docentes que diseñaron e implementaron propuestas (Figura 1) que abarcaron las áreas de Educación Ambiental; para la Salud Sexual; Física y Físico-química; Biología; y uso del laboratorio para abordajes experimentales de fenómenos fisicoquímicos y biológicos. En las JIPE se presentaron, entre otros, los análisis

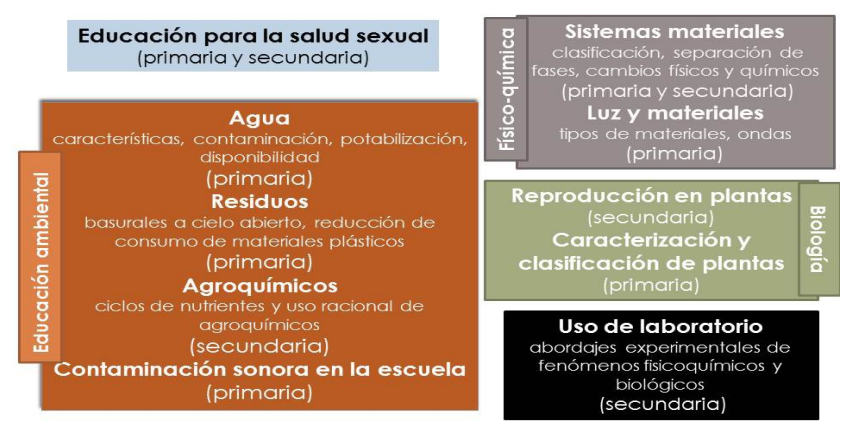
de las innovaciones realizados por 5 de estos grupos, luego recopilados en un Figura 1. Temáticas elegidas por las/os docentes para elaborar libro de autoría colectiva (Dumrauf, sus propuestas didácticas. Cordero y Mengascini 2013).

Las fuentes para la reconstrucción y fundamentación del enfoque de formación docente, que describimos en este artículo, fueron: registros de audio, fotográfico y filmaciones de encuentros de trabajo y reuniones; registros de clases implementadas en las escuelas, elaborados y disponibilizados por las/os docentes; y producciones de los grupos docentes durante el proceso. Los diversos registros fueron sistematizados, organizados y reducidos para el análisis de contenido (Bardin, 2002), en diversas instancias de profundización. El análisis aquí presentado constituye una revisión de características y fundamentos específicos del triple proceso sostenido.

\section{Características estructurantes de la experiencia formativa}

Identificar aspectos promotores de cambios en las prácticas de enseñanza de las/os docentes llevó a reconocer rasgos propios y originales del triple proceso de formación docente, transformación didáctica e investigación participativa (Figura 2). 
Respecto al proceso de formación, una característica especialmente valorada por las/os participantes fue plantear la vivencia de propuestas didácticas coherentes con las estrategias promovidas para sus proyectos de aula (enseñanza por proyectos, modalidad de taller, abordajes experimentales). Articulamos estas estrategias con un diseño flexible del proceso formativo, que partió del análisis y la discusión del componente político de las prácticas educativas (a través de sus finalidades y características adjudicadas al rol docente); y que incluyó, posteriormente, los intereses temáticos de las/os participantes. Incorporamos también, como contenido, la sistematización de experiencias educativas (Jara 2012) por considerarla una herramienta metodológica pertinente para encarar, desde las condiciones de trabajo docente, investigaciones participativas.

La constitución de grupos docentes, en función de intereses comunes y posibilidad de trabajo conjunto, fue condición de partida para la transformación de las prácticas y devino fortaleza de la propuesta. En el balance realizado al finalizar el proceso, los/las docentes valoraron el intercambio con otros docentes y el trabajo conjunto, los aportes y producciones surgidas durante la propuesta implementada; y la formación de grupos de trabajo. La conformación de grupos de trabajo posee además el potencial de consolidar colectivos que continúen en el tiempo.

La elección, por parte de las/os docentes, de las temáticas para diseñar propuestas didácticas dio lugar a cuestiones y preocupaciones "sentidas" en el ámbito escolar (Freire 1997). La formulación de dichas propuestas en términos de problemáticas locales o institucionales se logró al vincular los intereses iniciales con la reflexión sobre las finalidades de la educación científica, el rol docente y el interés docente por la participación estudiantil. Ello se enriqueció a partir de un ejercicio constante de problematización y del aporte de bibliografía específica, orientados a poner en contacto las problemáticas elegidas con la investigación educativa. La bibliografía, periódicamente ofrecida, permitió que las/os docentes se inspiraran para el diseño, reflexionaran y/o fundamentaran sus proyectos, sin realizar réplicas de investigaciones elaboradas en otros contextos, sino construyendo una articulación situada. La problematización interpeló sus producciones desde los enfoques conceptuales y políticopedagógicos tratados durante la formación, sin la intencionalidad de imponerlos.

La elaboración de propuestas didácticas se llevó a cabo en un marco de cambio educativo (nueva legislación nacional y Diseños Curriculares (DC) jurisdiccionales). El proceso formativo incluyó el análisis de los DC, favoreciendo su utilización en el diseño de propuestas. Estos DC habilitaban el abordaje de cuestiones hasta ese momento percibidas como no permitidas, como la Educación Sexual Escolar. Así, las intervenciones implementadas conjugaron las preocupaciones e intereses de las/los docentes y orientaciones de los DC. En tal sentido, el proceso se configuró como un camino de doble vía: la reforma curricular, determinada y estructurada en niveles de gestión, se entretejió con decisiones docentes orientadas a abordar problemáticas de sus prácticas. 
El análisis de las propuestas elaboradas evidenció tres dimensiones amplias de transformación de la práctica, emergentes en un entramado propio en cada proyecto: los saberes puestos en juego (su origen y cómo se accede a ellos), los roles de los sujetos participantes y las modalidades de trabajo implementadas en el aula. Las transformaciones más profundas implicaron la alteración simultánea de las tres dimensiones (como se describió en Dumrauf et al. 2013).

El proceso de investigación se desarrolló a través de dos modalidades diferenciadas. Una de ellas, de generación conceptual, apuntó a caracterizar las prácticas habituales de enseñanza de Ciencias Naturales a través de un cuestionario (analizado en Mengascini y Mordeglia 2014). Sus resultados se aportaron a la reflexión sobre la práctica en los encuentros de formación. La segunda modalidad de investigación, participativa, pretendió la elaboración conjunta de conocimientos y alternativas de acción con las/os docentes. Partir de problemáticas sentidas por las/os docentes implicó una alternativa motivadora, que acotaba la elección a cuestiones de su injerencia. Las formas de registro en las aulas fueron seleccionadas y puestas en práctica por ellas/os, sin intervención ni intromisión del equipo coordinador, a fin de estimular la colaboración intragrupal, respetar y valorar la autonomía docente.

Con relación al análisis, la generación de preguntas de investigación no evaluativas significó un cambio dificultoso para las docentes ${ }^{2}$. En función de dichas preguntas y preocupaciones evidenciadas en el proceso, el equipo coordinador asumió la tarea de proponer marcos de análisis pertinentes, cuya comprensión y utilización quedó a cargo de las docentes. La elaboración de producciones escritas que dieran cuenta del camino transitado resultó un último reto, que fue encarado de manera participativa. La estructura de presentación de los análisis fue la de "comunicación a congreso", característica del ámbito académico, pero prácticamente desconocida para las docentes. Esta estructura pretendió ser un facilitador, pero conllevó un nuevo aprendizaje en el que los grupos docentes precisaron del acompañamiento del equipo coordinador. Esta participación de las coordinadoras en la escritura no se reflejó en la firma de las producciones, a fin de destacar el papel fundamental de las docentes en todo el proceso, incluyendo la comunicación pública. Reconocíamos la importancia de la autoría para el fortalecimiento de la autoestima y la autovaloración como docentes y productoras de conocimientos.

\section{Fundamentos del enfoque de formación docente}

Como hemos dicho, a fin de delinear los fundamentos teórico-metodológicos del enfoque elaborado, estructuramos su presentación en torno a los elementos claves de la IAP definidos por Kemmis y McTaggart (2005): las características y el papel de lo colectivo; el rol de facilitación; el empoderamiento de los actores; y el dualismo investigación/acción. En su desarrollo, ejemplificamos algunos de los resultados alcanzados en el proceso a través de testimonios o producciones escritas de las protagonistas.

\section{Un colectivo para la participación y el tratamiento de problemáticas}

La democratización de las relaciones, el establecimiento de relaciones de participación no jerárquicas hacia el interior del colectivo, fue un horizonte de nuestra acción. Como tal, constituyó un proceso/desafío permanente, que alcanzamos en ciertos momentos hacia el interior del grupo coordinador y con algunos grupos de docentes. Más allá de su alcance parcial, el trabajo de construcción de la colaboración conformó simultáneamente el colectivo como totalidad interdependiente, heterogénea, más bien diversa y contradictoria.

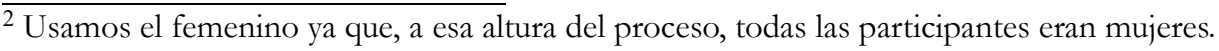


En nuestro caso, el triple proceso de formación, transformación de prácticas e investigación participativa requirió la creación de un colectivo de trabajo, conformado a su vez por grupos (equipo coordinador y grupos docentes), que permitió una cierta movilidad de sus in tegrantes y funcionó a modo de entramado de colaboración.

Existió diversidad en cuanto a objetivos en el inicio del trabajo conjunto. Las integrantes del equipo coordinador pretendíamos implementar y analizar un proceso de formación docente en ejercicio, que involucrara la investigación participativa. Intentábamos construir conjuntamente con docentes conocimiento que fuera un aporte a la resolución de problemas educativos. Aún a pesar de los esfuerzos para la difusión previa y precisa de la propuesta, los/as docentes participantes llegaron al primer encuentro de trabajo con la intención de asistir a una instancia de formación docente continua en el área de Ciencias Naturales, sin demasiada información sobre otros aspectos de la convocatoria. La formulación de objetivos compartidos, en torno al diseño, implementación y análisis de propuestas didácticas transformadoras, fue un camino que encontró obstáculos provenientes de esta diversidad inicial, de lógicas de trabajo diferentes y de posibilidades y habilitaciones personales e institucionales.

\begin{abstract}
"nosotras vinimos a hacer el proyecto por idea de la inspectora que nos impulsó a participar y mucho no entendíamos de qué se trataba todo esto y nos ha costado mucho llevar un hilo y ver qué era lo que se pretendía con esto de la innovación [...] Es como que uno está acostumbrado a presentar todo terminado y no todo el proceso." (DG8, 28/6/10) 3
\end{abstract}

En este camino emergieron también concreciones satisfactorias, realizaciones personales y grupales y nuevas posibilidades de acción. Tales realizaciones se vincularon, inicialmente, con el tratamiento de cuestiones que los grupos de docentes vivenciaban como insatisfacciones en su práctica cotidiana.

“trabajar así, significó un cambio de hábitos, moverse en el salón de un modo distinto, pararse muchas veces para buscar otros textos, tirarse en el piso para extender el afiche y escribir apoyados en el suelo, arrodillarse, preguntar cosas y buscar respuestas entre todos sin que sea la seño la que las dé.” (TPG7)

Las cuestiones trabajadas en las propuestas didácticas, al ser reconocidas, en la mayoría de los casos, como problemáticas sociales que involucran la ciencia y la tecnología (Massarini y Schnek 2015), permitieron también la identificación de situaciones injustas e irracionales (en el sentido habermasiano de no estar sustentadas en consensos racionales).

\footnotetext{
“[decidimos trabajar sobre] una problemática mundial y que por ende afecta a nivel local como lo es el uso indebido de las bolsas de polietileno, y su efecto nocivo sobre el medio ambiente [...] [porque] cuando los niños comienzan a preocuparse por las situaciones problemáticas que los rodean, se ven como agentes que entienden y son capaces de generar acciones para modificar y mejorar su entorno." (TPG7)

"Otra innovación para nosotras, que coincidía con el proyecto de las chicas, es que es mucho más interesante y más rico cuando el tema surge de una problemática que es de los chicos: la sequía y la falta de agua nos estaba pasando en ese momento y la estaban sufriendo ellos". (DG8, 28/6/10)
}

El tratamiento de estas problemáticas, que implicó cambios en la forma de conceptualizarlas, fue acompañado por novedades metodológicas que, entre otros aspectos, apuntaron a estimular la participación estudiantil, a nivel áulico y en el entorno local.

"El trabajo con proyectos cercanos a las vivencias de los alumnos permite salir de lo cotidiano, facilita el desarrollo de diferentes conocimientos y habilidades, los mantienen más activos, críticos y reflexivos. La literatura analizada aportó esta forma de trabajo, que no es muy frecuente en las aulas, pero que permitió al alumno ser el protagonista de sus aprendizajes." (TPG15)

\footnotetext{
${ }^{3}$ DG $n$ : docente del Grupo de Trabajo No $n$. TPG $n$ : texto extraído de producciones escritas del Grupo de Trabajo $\mathrm{N}^{\mathrm{o}} n$.
} 


\section{Roles de facilitación en la consolidación de colectivos}

La generación y sostenimiento del colectivo requirieron la asunción de roles que facilitaran su existencia. En nuestro caso, aunque nos nombrábamos como coordinadoras y no como facilitadoras, asumimos este rol, como también lo hicieron otros/as integrantes en diferentes momentos. Al comienzo, el conocimiento del proyecto, labores realizadas para su gestión y nuestros saberes específicos, colocaron al equipo coordinador frente a la tarea de facilitación del proceso. Hacia el interior de este equipo la división de roles entre las integrantes de la Universidad y del ISFD fue relativamente estable, con escasos desplazamientos. Si bien la planificación de los espacios de formación se consensuaba en las reuniones de equipo, a la hora de trabajar con las/os docentes, las tareas de coordinación, exposición teórica y síntesis conceptual eran asumidas por quienes proveníamos de la Universidad, mientras que las de organización, gestión y comunicación regional quedaban a cargo de las profesoras del ISFD. Sin embargo, una vez finalizado el Proyecto, estas profesoras evidenciaron aprendizajes y autoconfianza adquiridos a través de la experiencia, al ponerse al frente de nuevos proyectos institucionales y personales.

"Este año presentamos cinco proyectos de Feria [de Ciencias], para mí fue un gran éxito. Lograr que lleven a cabo una investigación, que hicieran un montón de cosas. Que por ahí llevábamos uno o dos, bueno, este año se hicieron cinco. Y bastante bien, pasaron tres a la Feria Regional [de Ciencias], creo que se ha logrado que los docentes intenten trabajar de otra manera y llevarlo a cabo con los chicos" (DG15, 12/9/13).

La facilitación también fue llevada a cabo por otros/as integrantes del colectivo: docentes de secundaria fueron facilitadores/as en la resolución de cuestiones conceptuales en grupos que incluían docentes de primaria; en grupos de maestras, un/a participante ejerció un rol de coordinación, comunicación y/o soporte grupal; y en otros casos, un miembro sostuvo la comunicación grupal con el equipo coordinador.

La asunción de estas tareas resultó clave para la construcción de este ámbito de colaboración. Boavida y Ponte (2002) entienden a la colaboración como el establecimiento de relaciones no jerárquicas, sobre una base de igualdad, de modo que se establezca ayuda mutua y objetivos que beneficien a quienes participan:

"Para mí lo mejor fue la relación que se estableció entre los docentes de la escuela y con ustedes [investigadoras de la Universidad]. Más allá de todo el trabajo que se hizo, de todo lo conceptual que sacamos, creo que la relación que se forjó entre docentes y con ustedes también tal vez fue lo más rico de todo lo que se hizo". (Integrante del equipo coordinador del ISFD, 12/9/13)

Para los autores antecitados la colaboración involucra la negociación cuidadosa, la toma conjunta de decisiones, la comunicación efectiva y el aprendizaje mutuo en un emprendimiento focalizado en la promoción del diálogo profesional.

\section{Toma colectiva de decisiones y empoderamiento}

La toma de decisiones respecto de los diversos procesos imbricados en nuestro enfoque de formación docente tuvo lugar en diferentes espacios colectivos, en los que la argumentación y búsqueda de consenso fueron las acciones orientadoras. La constitución interdisciplinar e interinstitucional del equipo coordinador permitió considerar diversas lógicas institucionales y diferentes saberes en la elaboración del proyecto. Este diseño tuvo tres características centrales: apertura para incluir los intereses temáticos de las/os cursantes; ejercicio de desnaturalización de lo cotidiano y socialización de alternativas teóricas y prácticas. Dicho ejercicio posibilitó fundamentar prácticas existentes y visualizar nuevas configuraciones, como aportes a la toma de decisiones legítimas. 
"haciendo lectura de la bibliografía [abordada en el proceso], nos dimos cuenta que muchas actividades propuestas por nosotras se enriquecerían inmensamente al ser trabajadas de esta manera [a través de la modalidad taller], se nos abrieron muchas opciones nuevas, empezamos a ver las actividades con otra mirada y tuvimos que reformular algunas cuestiones." (TPG11)

Cada etapa de la formación culminó con una socialización de resultados y evaluación conjunta de lo vivenciado, a fin de planificar los pasos a seguir, teniendo en cuenta las necesidades concretas de la práctica.

En el mismo sentido, los procesos de transformación de prácticas e investigación participativa se orientaron a la constitución de espacios colectivos para la toma de decisiones. Así, la toma de decisiones se transformó en un contenido implícito de la formación. Un primer espacio de este tipo fueron los grupos docentes de trabajo en los que se reconstruyó la propia práctica, se argumentó sobre sus fundamentos y características y se imaginó colectivamente cómo transformarla. En la encuesta de evaluación final del proceso, todas las docentes coincidieron en calificar este aspecto como altamente, muy satisfactorio o satisfactorio. Alguna especificó como aporte del proyecto:

"El intercambio con otros docentes y el trabajo conjunto, los aportes y producciones surgidas durante la propuesta implementada; formar un grupo de trabajo". (Encuesta de evaluación final del Proyecto)

Los encuentros formativos también constituyeron espacios colectivos de decisión, fundamentalmente en los momentos de puesta en común del proceso de diseño, implementación y análisis de las propuestas elaboradas. Los grupos de trabajo expusieron sus experiencias y recibieron comentarios, críticas y sugerencias de otros grupos y del equipo coordinador. Otro espacio de este tipo fueron los encuentros entre grupos docentes en sus instituciones y el equipo coordinador. Esta instancia exigió un proceso de construcción de confianza, a fin de superar los temores generados por las prácticas habituales de supervisión y evaluación de la tarea docente, y lograr la explicitación de dificultades propias del inicio de nuevos proyectos. Ambas instancias permitieron el afianzamiento de las decisiones tomadas y/o la reorientación de las intenciones de acción.

"Yo no había leído mucho de la colaboración y qué es colaborar ¿no? Y colaborar es el estilo que ustedes tienen de trabajo [...] La forma de trabajo que tienen a mí me sirve para trabajar en la escuela, ahora desde otro lugar, por ahí ya no en la actividad específica en el aula, pero sí de trabajar en equipo pero de manera colaborativa. Es decir, con un extremo respeto por la producción del otro y del aporte que hace al grupo y sentir ese apoyo incondicional.” (Directora ISFD, 12/9/13)

Por lo tanto, desde la propuesta de constitución de grupos docentes hasta la presentación pública de análisis sobre lo implementado en las aulas, cada etapa involucró la necesidad de fortalecimiento de la autoestima y reafirmación personal y grupal para la intervención transformadora y la participación discursiva en espacios cada vez más amplios de debate sobre las acciones y sus fundamentos. Así, la apropiación colectiva de la acción docente y el ejercicio del debate en nuevas esferas públicas habrían sido las bases para el empoderamiento de las/os participantes.

\section{Acciones exploratorias como superación del dualismo investigación-acción}

El trabajo desarrollado con las/os docentes en torno a sus propuestas didácticas se centró en explorar alternativas de intervención a través de su vivencia, reflexionando acerca del proceso y sus consecuencias, a fin de aprender de la propia experiencia:

"DG81: Como experiencia ha sido muy interesante...

DG82: Más que nada el replanteo de nuestro rol, como decían las chicas, esto de orientar y guiar y dejar que los chicos hagan y se pregunten, es mucho más productivo cuando uno los deja a ellos hacer, les da esa libertad. Nos dio mucho trabajo, todavía no lo hemos podido cerrar, creo que por ahí se nos abrió 
mucho en algún momento. Y después fuimos cerrándolo para limitarnos a una sola cosa y después poder hacer la conclusión sobre eso". (DG8, 28/6/10)

Según Fals Borda (1985, p. 129), "por la vivencia de una cosa [...] aprehendemos su realidad, sentimos, gozamos y entendemos los fenómenos cotidianos, y experimentamos nuestro propio ser en su contexto total". De acuerdo con nuestro análisis, la exploración de alternativas fue posible a partir de la vivencia de procesos formativos, de investigación participativa y de transformación didáctica sostenidos por lógicas diferentes respecto de las habituales en espacios de formación docente continua. Estas se caracterizan por la desconsideración de los saberes docentes, enfoques transmisivos y limitada participación en la toma de decisiones. La vivencia de otras lógicas, relaciones de poder y prácticas en el espacio formativo y en el de investigación, pretendió promover la transformación de lógicas y prácticas en sus espacios de trabajo docente.

"Yo creo que también fue animarse a hacer otras cosas. Es decir sentirse contenido cuando uno hizo algo que es diferente a lo que es habitual, aunque había cosas que uno habitualmente hacía, otras que no, la mirada del otro y la confianza que uno sentía, aunque a veces parecía que uno se iba para cualquier lado y ustedes nos encauzaban [risas], nos animó a hacer por ahí otras cosas, aunque a veces no en [Ciencias] Naturales, pero en otras áreas con los chicos. Perdés ${ }^{4}$ por ahí el miedo.” (DG8, 12/9/13)

Este ensayo de nuevas alternativas, estas acciones exploratorias, habilitaron a las participantes para la elaboración y ejecución de otras iniciativas. Ambos procesos incluyeron transitar diversas emociones: satisfacción, alegría, disfrute, y también miedo, inseguridad, dudas, como expresaron muchas docentes.

“A partir de nuestra propia práctica pudimos ir desterrando los miedos que teníamos al inicio del proyecto: ¿qué preguntarán los niños? ¿Estaré preparada para responderlas? ¿Las respuestas a sus dudas no los incentivarán a hacer cosas prematuras para su edad?” (TPG1)

La posibilidad de expresión y contención de los procesos emocionales necesitó además la construcción de un ámbito de confianza. Ello constituyó, como en toda propuesta colaborativa, un aspecto fundamental, ya que los/as participantes precisamos sentirnos con la libertad de expresarnos y de cuestionar abiertamente ideas, valores y acciones, a partir del respeto mutuo. Como dicen Boavida y Ponte (2002) la confianza se asocia a la disponibilidad de escucharse, a la valoración de las contribuciones y al sentimiento de pertenencia al grupo. A ello apuntaron las lógicas de trabajo adoptadas, pensadas como rupturas en el orden establecido que permitieran visualizar otras realidades posibles. Tales aspectos afectivos, necesarios de considerar en una visión integral de las personas, aportados por la conceptualización de Fals Borda (1985), complementan, a nuestro criterio, los elementos incluidos en las nociones de acción comunicativa y exploratoria en esferas públicas, propuestas por Kemmis y McTaggart (2005).

La participación en acciones exploratorias promovería aprendizajes plasmados en comprensiones colectivas, más que en conocimiento educativo teórico enunciable en términos convencionales (como aparece en la literatura educacional). Dichas comprensiones "se acumulan en conversaciones, archivos de evidencias y en el conocimiento compartido en comunidades de prácticas" (Kemmis y McTaggart 2005, p. 322, traducción nuestra). Y más aún, los resultados de la IAP producirían otras historias, para quienes participan, sus escuelas y las personas con quienes interactúan. El testimonio de una de las docentes al finalizar el proceso, intenta sintetizar los cambios vivenciados en sus prácticas:

"Los cambios se dieron en tres etapas: 1) con el grupo [de trabajo]: tomando confianza con el tema, con bibliografía adecuada y preparándome mucho antes de dar las clases; 2) con los registros: notando que los chicos aprendían, que se hablaba de ciencias y que lograba sostener el objetivo planteado; 3) con los

${ }^{4}$ Forma de conjugación utilizada en Argentina para la segunda persona del singular, equivalente al "Pierdes" español. 
análisis: a partir de descubrir los patrones temáticos [propuestos por Lemke 1997] y dilucidar que se establecían relaciones y que era capaz de analizar mi práctica, descubriendo errores y virtudes". (Encuesta de evaluación final del Proyecto).

\section{Reflexiones finales}

Las características y fundamentos presentados delinearon un enfoque para la formación docente en Educación en Ciencias Naturales, Ambiental y en Salud con múltiples facetas político-pedagógicas originales. Por un lado, permitió articular la investigación educativa con la realidad escolar a través de la producción conjunta de conocimientos. Por otro, promovió cambios en las prácticas docentes respecto de: la lectura de lo real (al plantear la definición de contenidos en torno a problemáticas sociales que involucran la ciencia y la tecnología); y las relaciones sociales (al estimular la construcción colectiva de propuestas y análisis y la participación estudiantil). Finalmente, valoró el papel de la praxis a través de la producción de acciones exploratorias. Todos estos cambios estuvieron orientados a transformar la realidad educativa desde una intencionalidad emancipatoria. Las múltiples facetas de esta construcción se articularon a partir de un elemento fundamental: la participación real de las/os docentes en todas las instancias de toma de decisiones en esferas públicas creadas a lo largo del proceso de trabajo. La creación y ampliación de dichas esferas se basó y contribuyó dialécticamente al progresivo empoderamiento y construcción de autonomía logrados por las/os participantes. Tales logros se manifestaron, además de en los cambios pensados y plasmados en sus prácticas docentes en el marco del proyecto (descritos en Dumrauf y Cordero 2018), en la elaboración y concreción de nuevas iniciativas personales y colectivas (como el desarrollo de Jornadas de Intercambio de Experiencias Educativas, organizadas anualmente por el ISFD a partir de 2012; el inicio de carreras universitarias relacionadas o de circuitos formativos de posgrado por parte de algunas de las protagonistas motivadas por lo aprendido en esta experiencia). También se reflejaron en diversas producciones analíticas de las propuestas educativas implementadas (entre otras: Guerra, Tetáz y Letché 2013; Castellá, Martínez y Marsiglia 2013; Castillo y Magnaterra 2013; Zubiarrain 2013; Asad y Suárez 2013; Martínez, Marsiglia, Herrero, Cordero, Mengascini y Dumrauf 2009; Dumrauf, Castellá, Cordero, Herrero, Fontana, Martínez, Marsiglia, Mengascini y Mordeglia 2009; Mengascini, Cordero, Marsiglia, Herrero y Dumrauf 2008).

Este enfoque pedagógico-didáctico, con las debidas adecuaciones, podría orientar otros procesos de formación docente a través de investigaciones participativas. Ello implicaría, dado su carácter situacional, adaptaciones a cada contexto, respetando la singularidad de personas y colectivos involucrados y sus intencionalidades.

\section{Referencias bibliográficas}

Achilli, E. (2004) Investigación y formación docente. Rosario: Laborde Editor.

Asad, N. y Suárez, M.R. (2013) La contaminación sonora afecta la salud. Análisis de nuestras prácticas para transformarlas. En A. Dumrauf, , S. Cordero y A. Mengascini (coords.), De docentes para docentes: experiencias innovadoras en ciencias naturales en la escuela pública (pp. 43-50). Buenos Aires: El Colectivo.

Bardin, L. (2002) Análisis de Contenido (Vol. 89). Madrid: Akal.

Boavida, A.M. y Ponte, J.P. (2002) Investigação colaborativa: Potencialidades e problemas. En GTI (org.), Reflectir e investigar sobre a prática profissional (pp. 43-55). Lisboa: APM.

Castellá, J.J., Martínez, M.C. y Marsiglia, M.R. (2013) El Museo Didáctico Escolar de Ciencias Naturales de la Escuela Normal de Dolores: una experiencia de innovación en la 
enseñanza de las Ciencias Naturales. En A. Dumrauf, S. Cordero y A. Mengascini (coords.), De docentes para docentes: experiencias innovadoras en ciencias naturales en la escuela pública (pp. 65-74). Buenos Aires: El Colectivo.

Castillo, I. y Magnaterra, F. (2013) Proyecto de innovación e investigación colaborativa: que nos quede claro... como el agua. En A. Dumrauf, S. Cordero y A. Mengascini (coords.), De docentes para docentes: experiencias innovadoras en ciencias naturales en la escuela pública (pp. 27-35). Buenos Aires: El Colectivo.

Copello Levy, M.I. y Sanmartí Puig, N. (2001) Fundamentos de un modelo de formación permanente del profesorado de ciencias centrado en la reflexión dialógica sobre las concepciones y las prácticas. Enseñanza de las Ciencias 19 (2), 269-283. https://www.raco.cat/index.php/Ensenanza/article/view/21741/21574

Cordero, S. y Mengascini, A. (2016) La construcción de una comunidad de práctica: articulaciones posibles entre la escuela y la academia. Cuadernos de la Facultad de $\begin{array}{lllll}\text { Humanidades } & y & \text { Ciencias } & \text { Sociales } & \text { 147-159. }\end{array}$ http://www.redalyc.org/pdf/185/18552439005.pdf

Delord, G., Porlán, R. y Harres, J. (2017) La importancia de los proyectos y redes innovadoras para el avance de la Enseñanza de las Ciencias: El caso de un profesor de la Red IRES. Revista Eureka sobre Enseñanza y Divulgación de las Ciencias 14 (3), 653-665. DOI: 10498/19514

Dumrauf, A. y Cordero, S. (2018) Tramas entre escuela y universidad. Formación docente, innovación e investigación colaborativa. La Plata: EDULP. http://sedici.unlp.edu.ar/handle/10915/69683

Dumrauf, A., Cordero, S. y Mengascini, A. (coords.) (2013) De docentes para docentes. Experiencias innovadoras en Ciencias Naturales en la escuela pública. Buenos Aires: Editorial El Colectivo.

Dumrauf, A., Castellá, J.J., Cordero, S., Herrero, M.G., Fontana, M.A., Martínez, C., Marsiglia, M.R., Mengascini, A. y Mordeglia, C. (2009) La investigación colaborativa: ¿una herramienta para transformar la educación científica? Actas de las II Jornadas de Enseñanza e Investigación Educativa en el campo de las Ciencias Exactas y Naturales. La Plata: UNLP. http://www.jornadasceyn2.fahce.unlp.edu.ar/actas/DUMRAUF\%2C\%20et $\% 20$ al. \%202009.pdf/

Fals Borda, O. (1985) Conocimiento y poder popular. Lecciones con campesinos de Nicaragua, México, Colombia. Bogotá: Siglo XXI editores de Colombia.

Feldfeber, M. (2011) "Los docentes como sujetos del campo pedagógico: una mirada desde las políticas educativas". En F. Hillert, N. Graziano, y M. J. Ameijeiras. La mirada pedagógica para el siglo XXI: teorías, temas y prácticas en cuestión: reflexiones de un encuentro (pp. 103-119). Buenos Aires: Editorial de la Facultad de Filosofía y Letras, UBA.

Freire, P. (1997) Pedagogía de la autonomía. México: Siglo XXI editores.

Guerra, A., Tetáz, B. y Letché, M. (2013) "Proyecto de innovación en educación ambiental: por una vida mejor". En A. Dumrauf, S. Cordero y A. Mengascini (coords.), De docentes para docentes: experiencias innovadoras en ciencias naturales en la escuela pública (pp. 35-42). Buenos Aires: El Colectivo.

Habermas, J. (1984) Theory of communicative action, Vol. 1: Reason and the rationalization of society. Boston: Beacon.

Habermas, J. (1996) Between Facts and Norms. Cambridge, Massachusetts: MIT Press. 
Hoyos Vásquez, G. (1998) Introducción. De la investigación-acción participativa a la teoría de la acción comunicativa. En G. Hoyos Vásquez y A. Uribe (comps.), Convergencia entre ética y política (pp. 1-15). Bogotá: Siglo del Hombre Editores.

Jara, O. (2012) La sistematización de experiencias. Práctica y teoría para otros mundos posibles. San José, C.R.: Centro de Estudios y Publicaciones Alforja, CEAAL, Intermon Oxfam.

Kemmis, S. y McTaggart, R. (2005) Participatory action research: Communicative action and the public sphere. En N.K. Denzin y Y.S. Lincoln (eds.), The Sage Handbook of Qualitative Research (pp. 559-603). California: Sage Publications.

Lemke, J. (1997) Aprender a bablar ciencia. Lenguaje, aprendizaje y valores. Buenos Aires: Paidós

Loureiro, C.F.B. (2003) Emancipación, complejidad y método histórico dialéctico: repensar las tendencias en educación ambiental. Tópicos en Educación Ambiental 5(13), 21-30. http://www.anea.org.mx/Topicos/T\%2013/Paginas\%2021\%20-\%2030.pdf

Lupión Cobos, T. y Martín Gámez, C. (2016) Desarrollo profesional docente de profesorado de secundaria en una experiencia de innovación mediante investigaciones escolares. Revista Eureka sobre Enseñanza y Divulgación de las Ciencias 13 (3), 686-704. http://dx.doi.org/10.25267

Martínez, C., Marsiglia, M.R., Herrero, G., Cordero, S., Mengascini, A. y Dumrauf, A. (2009) Innovación y trabajo colaborativo en la enseñanza de las Ciencias Naturales: una experiencia didáctica sobre los dilemas éticos del proyecto genoma humano. Revista Iberoamericana de Educación 8 (4), 2-11. https:/ /doi.org/10.35362/rie4842180

Massarini, A. y Schnek, A. (2015) Ciencia entre todxs. Tecnociencia en contexto social. Una propuesta de enseñanza. Buenos Aires: Paidós.

Mengascini, A. y Mordeglia, C. (2014) Caracterización de prácticas experimentales en la escuela a partir del discurso de docentes de primaria y secundaria. Enseñanza de las Ciencias 32 (2), 71-89. http://dx.doi.org/10.5565/rev/ensciencias. 755

Mengascini, A., Cordero, S., Marsiglia, M.R., Herrero, G. y Dumrauf, A. (2008) Palabras desde el camino: Reconstrucción de una experiencia de investigación colaborativa en educación en ciencias naturales. Actas Electrónicas de los XXIII Encuentros de Didáctica de las Ciencias Experimentales (pp. 633-642). Almería (España). http://apicedce.com/2018/08/08/actas-de-los-xxiii-encuentros-de-didactica-de-las-cienciasexperimentales/

Menghini, R., Díaz, M., Gardié, S. e Iriarte, L. (2011) Política curricular de formación de docentes en la Provincia de Buenos Aires. En L. Porta, Z. Álvarez, , C. Sarasa, y S. Bazán (coords.), Actas de las VI Jornadas Nacionales sobre la Formación del Profesorado (pp. 114). Mar del Plata: UNMdelP.

Messina, G. (1999) Investigación en o investigación acerca de la formación docente: un estado del arte en los noventa. Revista Iberoamericana de Educación 19, 145-207. https://doi.org/10.35362/rie1901057

Oliva, J.M. (2011) Dificultades para la implicación del profesorado de Educación Secundaria en la lectura, innovación e investigación en didáctica de las ciencias (I): el problema de la inmersión. Revista Eureka sobre Enseñanza y Divulgación de las Ciencias 8(1), 41-53. https://revistas.uca.es/index.php/eureka/article/view/2692/2341

Oliva, J.M. (2012) Dificultades para la implicación del profesorado de Secundaria en la lectura, innovación e investigación en didáctica de las ciencias (II): el problema del "manos a la 
obra". Revista Eureka sobre Enseñanza y Divulgación de las Ciencias 9(2), 241-251. https://rodin.uca.es/xmlui/bitstream/handle/10498/14732/5-274-Oliva.pdf? sequence $=6$

Pacca, J.L. A., Villani, A. (2000) La competencia dialógica del profesor de ciencias en Brasil. $\begin{array}{lllll}\text { Enseñanza de las } & \text { Ciencias } & 18 & \text { (1), } & \text { 95-104. }\end{array}$ https://www.raco.cat/index.php/Ensenanza/article/view/21642/21475

Tardif, M., Zourhlal, A.A. (2005) Difusão da pesquisa educacional entre profissionais do ensino e círculos acadêmicos. Cadernos de Pesquisa 35 (125), 13-35. http://www.scielo.br/pdf/cp/v35n125/a0335125.pdf

Vázquez-Bernal y Jiménez Pérez, R. (2013) Un modelo de innovación en el Practicum de Secundaria: la inmersión dentro de un grupo de investigación-acción. Revista Eureka sobre Enseñanza y Divulgación de las Ciencias 10 (Núm.extraordinario), 709-727. DOI: http://dx.doi.org/10.25267/Rev_Eureka_ensen_divulg_cienc.2013.v10.iextra.15

Zeichner, K.M. (1998) Para além da divisão entre professor-pesquisador e pesquisador acadêmico. En C.M. Geraldi, D. Fiorentini y E.M.A. Pereira (orgs.), Cartografias do trabalho docente: professor(a)-pesquisador(a) (pp. 207-236). Campinas, Mercado das Letras: Associação de Leitura do Brasil.

Zubiarrain, L. (2013) Hacia una decente práctica docente. En A. Dumrauf, S. Cordero y A. Mengascini (coords.), De docentes para docentes. Experiencias innovadoras en Ciencias Naturales en la escuela pública (pp. 51-64). Buenos Aires: El Colectivo. 